\title{
Influence of fiber parameters to the erbium-doped fiber amplifier (EDFA) gain: A theoretical modeling
}

\author{
N. A. B. Ahmad ${ }^{1,2, *}$, S. H. Dahlan ${ }^{1,2}$, and N. A. Cholan ${ }^{1,2}$ \\ ${ }^{1}$ Research Centre for Applied Electromagnetics, Universiti Tun Hussein Onn Malaysia (UTHM), Batu Pahat, Johor, Malaysia. \\ ${ }^{2}$ Faculty of Electrical and Electronic Engineering, Universiti Tun Hussein Onn Malaysia (UTHM), Batu Pahat, Johor, Malaysia.
}

\begin{abstract}
This paper studies the theoretical analysis of erbium-doped fiber (EDF) amplification. The EDF amplification is investigated in order to understand its behavior and identify the relevant parameters that are used for formulating the amplification process related to spontaneous and stimulated emission. Results show that under certain condition, the selected fiber parameters such as fiber length, EDF pump power, signal power and pump direction would significantly contribute to the EDFA gain enhancement.
\end{abstract}

\section{Introduction}

Multiwavelength fiber lasers have attracted great interest due to their applications in optical sensors, microwave optics, spectroscopy and optical communication systems to name a few. One technology where these fiber lasers can be realized is by a combination of two optical gains namely erbium-doped fiber (EDF) and Brillouin amplification or commonly known in literature as multiwavelength Brillouin-erbium fiber lasers (BEFLs). Compared with other technologies, multiwavelength BEFLs have advantages such as low threshold power and ultra-narrow linewidth [1]. Literature suggests that performances of multiwavelength BEFLs can be improved when the residual waves are recycled back into the laser cavity [2]. Characteristics of residual waves are studied thoroughly in [3-4] to gain higher number of channels. This paper intents to optimize the EDF amplification in the multiwavelength BEFLs to improve the performance further. Therefore in this work, erbiumdoped fiber amplifier (EDFA) parameters for different pumping powers and different EDF length are investigated to realize a high performance EDFAs.

\section{Mathematical Modelling of EDFA}

The basic configuration of a typical EDFA is illustrated in Fig. 1. An EDFA consists of a wavelength division multiplexer (WDM) coupler to combine the pump with the input signal light. The combined pump and signal light is injected into a $20 \mathrm{~m} \mathrm{EDF}$ and an optical isolator is placed after the EDF, ensuring unidirectional operation of the EDFA. The pump source is of a $980 \mathrm{~nm}$ laser diode.

Fig. 2 illustrates the energy levels and transitions of $\mathrm{Er}^{3+}$ ions in the EDF when it is pumped with the $980 \mathrm{~nm}$ laser diode. It is based on a two-level assumption. At first, the ions absorb the pump power, enabling them to transit to the high level. The ions stayed at the level for a period of $\tau_{3}$ before jumping to the meta-stable state, resulting in emission of photons [5]. Due to the small value of $\tau_{3}$, it can be neglected and hence simplifies the three-level system to the two-level [6]. A radiative transition towards the ground energy level then takes place. As a result, photons at $1550 \mathrm{~nm}$ are emitted. The rate equations, eqs. (1) - (3) describe the effects of absorption, stimulated and spontaneous emission on the ions populations of the ground state $N_{1}$ and metastable states $N_{2}[7-8]$

$$
\begin{gathered}
\frac{d N_{1}}{d t}=-\left[W_{s a}(z)+W_{P}(z)\right] N_{1}+\left[\frac{1}{\tau_{21}}+W_{s e}(z)\right] N_{2}, \\
\frac{d N_{2}}{d t}=\left[W_{s a}(z)+W_{P}(z)\right] N_{1}-\left[\frac{1}{\tau_{21}}+W_{s e}(z)\right] N_{2}, \\
N_{1}(z)+N_{2}(z)=N_{T}
\end{gathered}
$$

where $\tau_{21}$ is the spontaneous emission lifetime, $N_{T}$ is the dopant density of the EDF, $W_{s a}, W_{s e}$, and $W_{P}$ are the stimulated absorption, stimulated emission and pumping rates respectively. Further elaborations can be found in [9] and summarizes as

$$
\begin{gathered}
W_{s a}(z)=\sum_{v} \frac{\xi_{s a}(v)}{A_{e f f} h v}\left[P_{S}+P_{A S E}^{+}(z, v)+P_{A S E}^{-}(z, v)\right] \eta(v) d v, \\
W_{s e}(z)=\sum_{v} \frac{\xi_{s e}(v)}{A_{e f f} h v}\left[P_{S}+P_{A S E}^{+}(z, v)+P_{A S E}^{-}(z, v)\right] \eta(v) d v, \\
W_{P}(z)=\frac{1}{A_{e f f}} \frac{\xi_{p a}\left(v_{P}\right)}{h v_{P}}\left[P_{P}^{+}(z)+P_{P}^{-}(z)\right] \eta\left(v_{P}\right),
\end{gathered}
$$

where $P_{S}$ is the signal power, $P_{P}^{+}$and $P_{A S E}^{+}$represent the forward propagating of EDF pump powers and amplified spontaneous emission (ASE) powers at frequency $v$, respectively, while $P_{P}^{-}$and $P_{A S E}^{-}$are the backward propagating of EDF pump powers and ASE powers at frequency $v$, correspondingly, $\xi_{s a}, \xi_{p a}, \xi_{s e}$, and $\xi_{p e}$ are

\footnotetext{
*Corresponding author: nurulati89@gmail.com
} 
the absorption and emission cross sections at the pump and signal wavelength. $A_{\text {eff }}$ is the effective area of the EDF and $h$ is the Plank's constant. Meanwhile, $\eta(v)$ is the overlapping factor between each radiation and fiber fundamental modes.

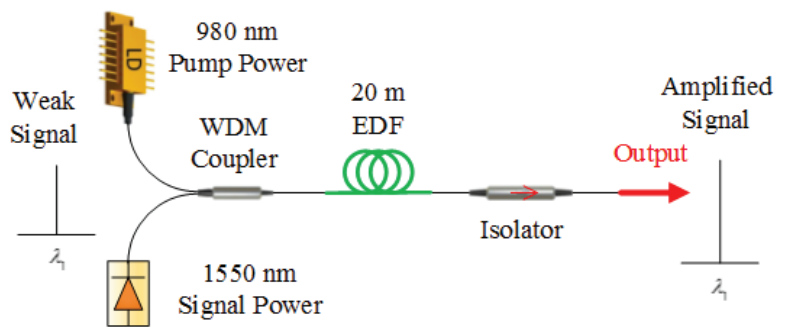

Fig. 1. Basic configuration of the EDFA.

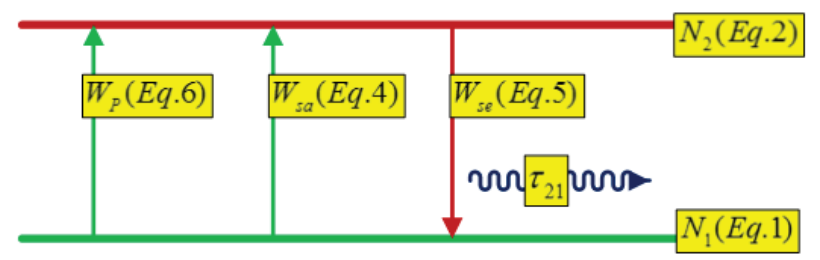

Fig. 2. Energy level diagram of $\mathrm{Er}^{3+}$ doped in silica.

Propagation of light through the EDF is shown in Fig. 3(a). The light in the EDF is assumed to propagate as a number of optical beams of frequency bandwidth $\Delta v$. The wave propagation of signal $P_{S}$, pump $P_{P}^{ \pm}$and noise power $P_{A S E}^{ \pm}$can be expressed as [10]

$$
\begin{gathered}
\frac{d P_{S}(z)}{d z}=\left(N_{2} \xi_{s e}-N_{1} \xi_{s a}\right) \eta\left(v_{S}\right) P_{S}(z), \\
\frac{d P_{P}^{ \pm}(z)}{d z}= \pm\left(N_{2} \xi_{p e}-N_{1} \xi_{p a}\right) \eta\left(v_{P}\right) P_{P}^{ \pm}(z),
\end{gathered}
$$

$\frac{d P_{A S E_{-} n}^{ \pm}(z)}{d z}= \pm\left[N_{2} \xi_{s e}(v)-N_{1} \xi_{s a}(v)\right] \eta(v) P_{A S E_{-} n}^{ \pm}(z) \pm d$,

where $d=2 h v \Delta v N_{2} \eta(v) \xi_{s e}(v)$ is the ASE power caused by the spontaneous emission.

In order to solve for power from the eqs. (1) - (6). the EDF with length $z$ is divided into segments along the forward and backward propagation as illustrated in Fig. 3(b). Each segment contains its own population densities. The pump, signal and ASE powers which propagate in the first segment (segment 0 ) are solved by using the initial condition as shown in Table 1. Then, the power of the earlier segment is used as the input for the next consecutive segments. The eqs. (7) - (9) are numerically solved through a trial and error technique known as relaxation method. This method makes iterative adjustment to the solution where an initial set of boundary is chosen for the first integration. Utilizing this method, accuracy of $0.01 \%$ is achieved for $P_{S}, P_{P}^{ \pm}$, and $P_{A S E}^{ \pm}[11-12]$.

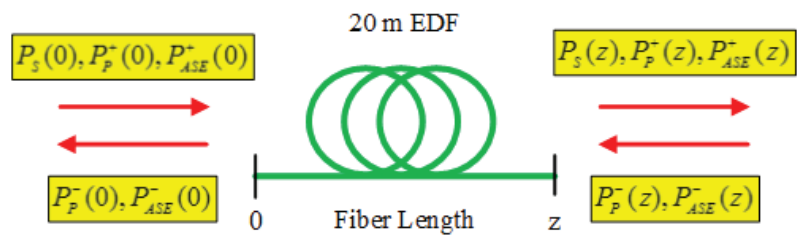

(a)

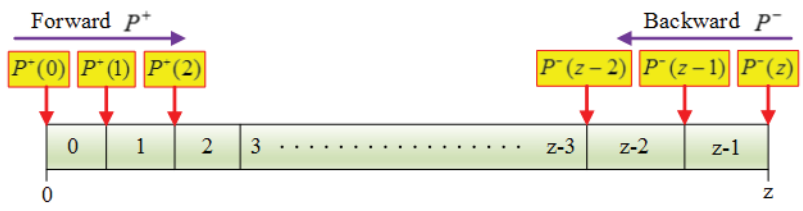

(b)

Fig. 3. (a) Lightwave propagation along the EDF. (b) Schematic of the numerical fiber model.

Table 1. Initial condition.

\begin{tabular}{|l|c|}
\hline \multicolumn{1}{|c|}{ Initial condition } & Operating wavelength (nm) \\
\hline$P_{P 1}(0)=P_{P}$ & $\lambda=980$ \\
\hline$P_{S}(0)=P_{S}$ & $\lambda=1550$ \\
\hline$P_{A S E}^{+}(0, v)=P_{A S E}^{-}(z, v)=0$ & $1450 \leq \lambda \leq 1600$ \\
\hline$P_{A S E}^{+}(z, v)=P_{A S E}^{-}(0, v) \neq 0$ & $1450 \leq \lambda \leq 1600$ \\
\hline
\end{tabular}

\section{Results and Discussions}

A series of numerical simulations are performed for the EDFA modelling using parameters of an EDF from OFS Model MP980. These parameters are $N_{T}=3 \times 10^{23}$, $N_{\text {2_initial }}=10^{-10}, L_{E D F}=20 \mathrm{~m}, \Delta v=5 \times 10^{10} \mathrm{~Hz}$, $\tau_{21}=10^{-2} \mathrm{~s}, \xi_{p a}=0, \xi_{p e}=3.28 \times 10^{-25} \mathrm{~m}^{2}, A_{\text {eff }}=50$ $\mu \mathrm{m}^{2}, c=3 \times 10^{8} \mathrm{~m} / \mathrm{s}$, and $h=6.63 \times 10^{-34} \mathrm{Js}$.

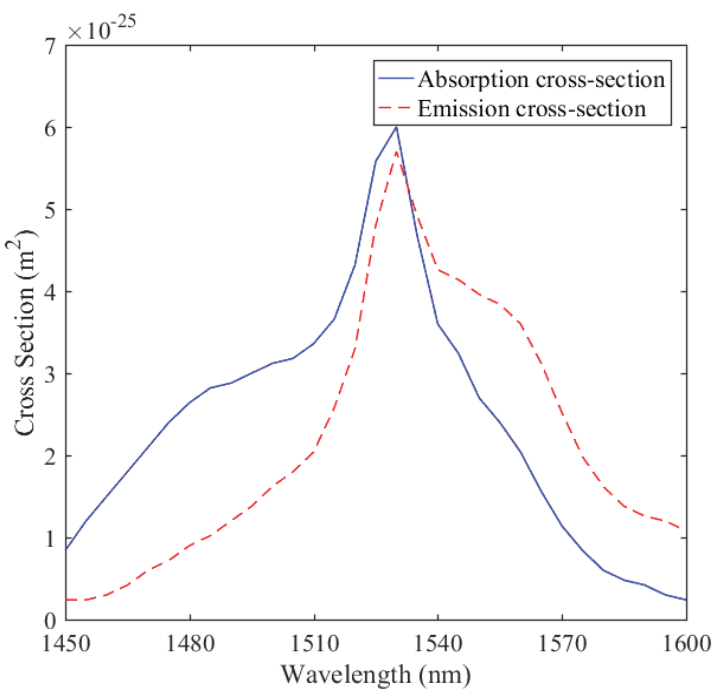

Fig. 4. Absorption and emission cross sections spectra of ASE signals. 
The $\xi_{s a}$ and $\xi_{s e}$ for the EDF of different wavelengths are obtained by using curve fitting method as shown in Fig. 4. Notice that each wavelength has its own absorption and emission coefficients since photons can be absorbed to generate carriers via a stimulated absorption process while at the same time, new photons can be generated owing to the stimulated emission process. Fig. 5 depicted the ASE power of both backward and forward ASE along the EDF for forward pumping configurations. During the simulation, we found that $P_{A S E}^{-}(z)$ must be lower than $0.01 \mathrm{~mW}$.

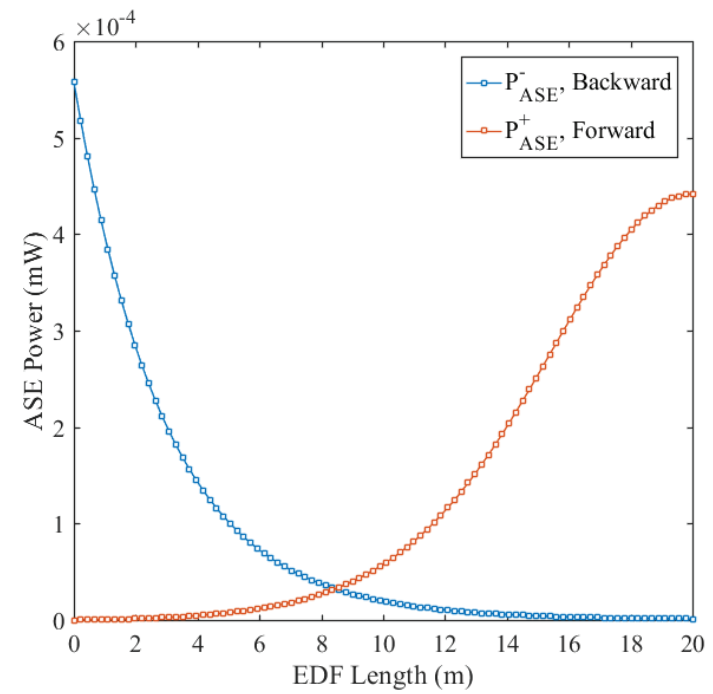

Fig. 5. Simulation output of forward and backward ASE.

The variation of gain, $G=P_{S}(z) / P_{S}(0)$ for different EDFs length and pump power is then investigated as shown in Fig. 6. With the EDF pump power increased from $30 \mathrm{~mW}$ to $100 \mathrm{~mW}$ for $20 \mathrm{~m} \mathrm{EDF}$, the gain in Fig. 6(a) was observed to increase along with the increasing EDF length. However, the gain started to decrease after reaching a maximum value. The reason is that the pump propagates through the EDF gets absorbed and hence the power reduces. After propagating a certain distance, the power becomes too small to create population inversion. As a result, the EDF would start to absorb the signal rather than amplify the signal. Therefore, there is an optimum EDF length to achieve maximum gain with variation of EDF pump power. Fig. 6 (b) depicted the variation of gain for different EDF length with the EDF pump power of $100 \mathrm{~mW}$. For EDF length from $10 \mathrm{~m}$ to $20 \mathrm{~m}$, the gain is expected to increase as the EDF pump power increase. When the EDF pump power increase, the population inversions are created gradually. No more $\mathrm{Er}^{3+}$ ions are available when all the $\mathrm{Er}^{3+}$ ions are excited, and thus the gain would saturate.

Further we study the EDFA gain by plotting EDFA gain versus wavelength with varying signal power from $40 \mathrm{dBm}$ to $-10 \mathrm{dBm}$ as depicted in Fig. 7. The results were obtained using a 20 m OFS MP980 of EDF and a $100 \mathrm{~mW}$ pump power at $980 \mathrm{~nm}$ wavelength. Initially, the gain spectrum of an EDFA is not uniform across the bandwidth. A narrow gain peak around $1530 \mathrm{~nm}$ and a relatively flat region between $1540 \mathrm{~nm}$ and $1560 \mathrm{~nm}$ can be observed in Fig. 7. When the input signal power increased from $-40 \mathrm{dBm}$ to $-10 \mathrm{dBm}$, the optical gain at
$1550 \mathrm{~nm}$ decreases from $37 \mathrm{~dB}$ to $15 \mathrm{~dB}$, resulting by gain saturation. Apart from that, the spectral shape of the optical gain also changes with the input signal power. When the input signal power increase, the gain tends to tilt toward the longer wavelength side while the short wavelength peak around $1530 \mathrm{~nm}$ is suppressed. This situation has to be considered in designing gainflattening techniques.
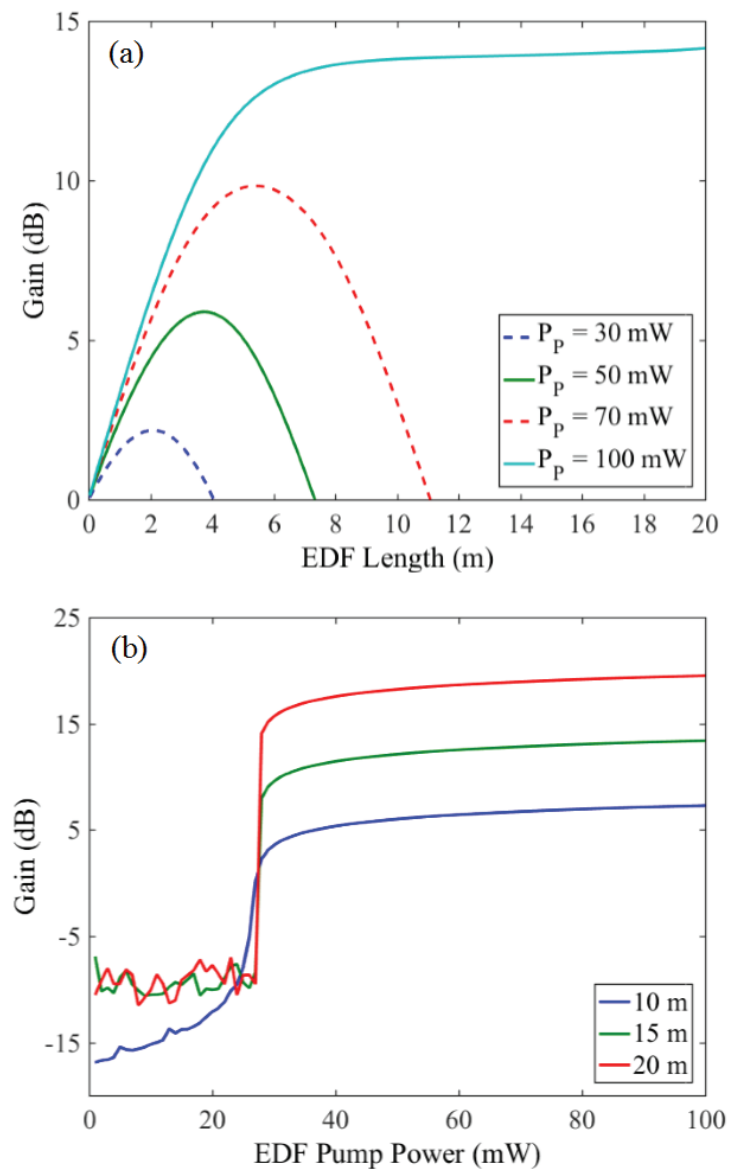

Fig. 6. The variation of gain with (a) EDF length and (b) Pump power

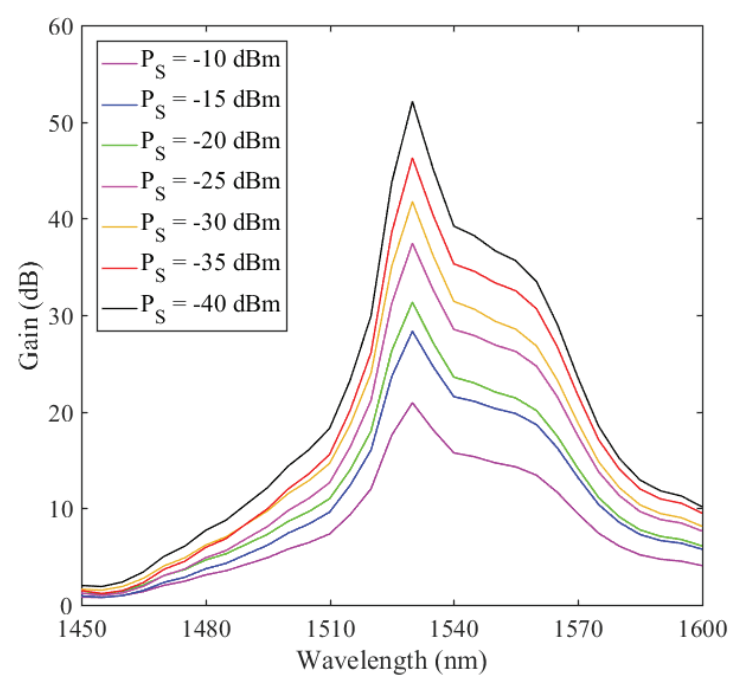

Fig. 7. EDFA gain at different input signal power with fixed EDF length of $20 \mathrm{~m}$ and pump power of $100 \mathrm{~mW}$ 


\section{Conclusion}

We have theoretically studied the effect of EDF parameters to the gain performance of C-band EDFAs. Theoretical analysis and simulation are carried out using the coupled rate and propagation equations, taking into accounts both the ground and excited states. The results show that the gain of EDFA is strongly dependent on the EDF length, pump power and input signal. When the pump power is sufficient enough, the EDFA shows that it could be operated in saturation regimes, leading to the maximum gain.

This work was supported in part by Fundamental Research Grant Scheme (FRGS) Vot 1622 and Research Acculturation Collaborative Effort (RACE) Vot 1509. Both grants are granted by the Ministry of Higher Education (MOHE) Malaysia. This work as well supported in part by Centre for Graduate Studies Universiti Tun Hussein Onn Malaysia (UTHM).

\section{References}

1. M. Chen, Z. Meng, Y. Zhang, J. Wang, W. Chen, IEEE Photonics J., 7, 7029071 (2015)

2. J. Tang, J. Sun, L. Zhao, T. Chen, T. Huang, Y. Zhou, Opt. Express, 19, 14682-14689 (2011)

3. N.A. Cholan, M.H. Al-Mansoori, A.S.M. Noor, A. Ismail, M.A. Mahdi, Opt. Comm., 329, 163-167 (2014)

4. N.A.B. Ahmad, S.H. Dahlan, N.A. Cholan, 2016 IEEE 6th International Conference on Photonics (ICP), 7510021 (2016)

5. O. De Varona, M. Steinke, D. Kracht, J. Neumann, P. Wessels, Opt. Express, 24, 24883-24895 (2016)

6. K.S. Thyagarajan, A. Ghatak, John Wiley \& Sons, 10 (2007)

7. M. Li, K. Liu, W. Jing, G.-D. Peng, J. Opt. Soc. Korea, 14, 14-21 (2010)

8. Y. Yuan, Y. Yao, J.J. Xiao, Y. Yang, J. Tian, C. Liu, J. Appl. Phys.,115, 043102 (2014)

9. X. Dong, P. Shum, N.Q. Ngo, H.-Y. Tam, X. Dong, J. Lightwave Technol., 23, 1334-1341 (2005)

10. S.D. Emami, H.A. Abdul-Rashid, H. Ahmad, A. Ahmadi, S.W. Harun, Ukr. J. Phys. Opt., 13, 74-81 (2012)

11. R. Hui, M. O’Sullivan, Academic Press, (2009)

12. T. Kasamatsu, Y. Yano, T. Ono, J. Lightwave Technol., 20, 1826-1838 (2002) 